\title{
ATTRIBUTIONS FOR CHANGE AMONG YOUNG ADULT BINGE DRINKERS
}

\author{
Youkyung Hwangbo ${ }^{1}$, Rachel Pace², Allison Wallace², James MacKillop³, \\ \& James Murphy ${ }^{2}$ \\ ${ }^{1}$ Katholische Universität Eichstätt-Ingolstadt (Germany) \\ ${ }^{2}$ University of Memphis (United States of America) \\ ${ }^{3}$ McMaster University (Canada)
}

\begin{abstract}
Introduction: Problem drinking has been shown to increase through adolescence, peak from ages 18-22, and decrease thereafter (Jackson \& Sartor, 2016). Research on natural recovery suggests that reasons for reduction may be associated with factors such as social capital, marital status, parenthood, employment, and religious and academic involvement (Vik, Cellucci \& Ivers, 2003; Dawson, Grant, Stinson \& Chou, 2006; Misch, 2007; Lee \& Sher, 2018).

Methods: The current study analyzed the 8-month follow up data of 444 participants from Project BETA, an ongoing study aimed at longitudinally examining trajectories of alcohol misuse in emerging adults (ages 21.5-24.99 at enrollment) over the course of 3 years. Participants were $57.3 \%$ female, $46.4 \%$ White, and $42.3 \%$ Black. They reported the change in their drinking level over the past month and the extent to which a variety of factors helped them to change their drinking. Furthermore, change in self-reported drinking was assessed in relation to the specific factors participants had selected.

Results: Regarding drinking level, $15.7 \%$ reported an increase, $40.4 \%$ reported no change and $43.8 \%$ reported a decrease. The most prevalent factors endorsed for decreased drinking levels were change in employment situation (55.9\%), change in diet to eat healthier foods $(49.7 \%)$, limiting access to money (47.7\%), change in financial situation (46.7\%), and change in social group (44.6\%). Chi square analyses were conducted for gender, race, alterations in drinking level and factors associated with the change. Independent samples t-tests were conducted to compare the change in average drinks per week from baseline to 8-months for White and Black participants in association with the specific factors they had selected.

Discussion: Further research is needed to investigate how drinking behavior can be influenced if participants are given accurate feedback on their level of drinking and the efficacy of factors associated with changes in that target behavior.
\end{abstract}

Keywords: Binge drinking, young adult, natural recovery, factors for changes in drinking level, differences between genders and ethnicity.

\section{Introduction}

An estimated $38.4 \%$ of young adults aged 18-25 reported binge alcohol use in the last 30 days (Substance Abuse and Mental Health Services, 2017). This is problematic yet unsurprising, as problem drinking has been shown to increase through adolescence, peak from ages 18-22, and decrease thereafter (Jackson \& Sartor, 2016). Research on natural recovery suggests that reasons for reduction may be associated with factors such as social capital, marital status, parenthood, employment, and religious and academic involvement (Vik, Cellucci \& Ivers, 2003; Dawson, Grant, Stinson \& Chou, 2006; Misch, 2007; Lee \& Sher, 2018).

Sociodemographic characteristics such as gender and ethnicity can play a significant role in risk for alcohol use and alcohol use disorders. Caucasian among different ethnicities has a superior tendency to be associated with alcohol abuse and dependence. Dependence on drinking alcohol took less time for both female and male Caucasians compared to African Americans (Alvanzo, Storr, La Flair, Green, Wagner \& Crum, 2011). Not only shown in ethnicities, but also in respect to gender, males have higher chances to consume excess alcohol and develop alcohol dependence than females (Wilsnack, Wilsnack, Kristjanson, Vogeltanz-Holm \& Gmel, 2009; Alvanzo, Storr, La Flair, Green, Wagner \& Crum, 2011).

The current study uses the data from Project BETA, which mainly applies behavioral economics to predict alcohol trajectories during the transition to adulthood. This study mainly focuses on changes drinking level between baseline and 8 months; moreover, the differences between genders and ethnicities, predominantly white and black within various factors of drinking behavior will be investigated. 


\section{Method}

\subsection{Participants}

A total of 602 Project BETA participants were enrolled at baseline however, our sample was limited to those who provided responses to the change in drinking measure at the 8-month follow up $(\mathrm{n}=444)$, which asked, "Over the past month has your overall drinking level changed?". The participants were between 21.5-24.99 years old at enrollment, and ethnicity of the participants was fairly equally divided between white and black as well as student or non-student. All participated sample were diagnosed as binge drinker which is defined as four or more drinks for women and five or more for men on one occasion.

\subsection{Procedure}

Participants were administered a computer questionnaire at baseline and at subsequent occasions every 8 months. Among them, only baseline and 8-month follow up were analyzed. The questionnaire gathered basic information about the participants such as age, gender and ethnicity, and drinks per week measured with the Daily Drinking Questionnaire (DDQ; Collins, Parks, \& Marlatt, 1985). Self-reported subjective change in drinking level was assessed at 8 month follow-up, with answers ranging from increased substantially to decreased substantially. Chi square analyses were conducted for gender, race, alterations in drinking level and factors associated with the change. Independent samples t-tests were conducted to compare the change in average drinks per week from baseline to 8-months for White and Black participants in association with the specific factors they had selected.

\section{Result}

We first compared the baseline and 8-month follow-up self-reported drinking level, with responses of Decreased Substantially, Decreased Moderately, Stayed the Same, Increased Moderately, and Increased Substantially. Based on average drinks per week, the percentage change was calculated.

If any change in drinking was self-reported, participants were then presented with 23 factors and instructed to select whether or not the factor played a role in the change. The top ten factors endorsed as contributing to increased levels of drinking were: increased stress/ anxiety $(67.1 \%)$, positive mood, more celebrations $(54.3 \%)$, change in your financial situation $(50.0 \%)$, increased access to alcohol $(48.6 \%)$, changing your living situation(44.3\%), increased sadness $(44.3 \%)$, boredom, inability to participate in hobbies or things you used to enjoy(41.4\%), relationship or family issues/ conflict(41.4\%), change in your employment situation(40.0\%), and increased craving(38.6\%). The factors were analyzed for differences gender and ethnicity. On average, participants selected 12 factors as associated with their increasing their drinking level. Males selected on average 8 different factors while females selected 13 factors on average. Black participants (16 factors) selected twice as many factors as White participants (8 factors). As a factor that associated with increased drinking, females were more likely to select change in financial situation $\left(\chi^{2}(1)=11.20, \mathrm{p}=.001\right)$ than males. Comparing to different ethnicity, the factors which are Increased Sadness $\left(\chi^{2}(1)=6.901, p=.009\right)$, Change in Living Situation $\left(\chi^{2}(1)=8.276\right.$, $\mathrm{p}=.004)$ and Change in Employment Situation $\left(\chi^{2}(1)=6.90, \mathrm{p}=.009\right)$ were shown significant differences. Top ten factors associated with decreased drinking were change in your employment situation(55.9\%), changing your diet to eat healthier foods(49.7\%), limiting access to money(47.7\%), change in your financial situation(46.7\%), change in your social group(44.6\%), change in exercising(41.0\%), changing your living situation(39.5\%), developing a new hobby or reinvesting in previous hobbies(39.0\%), practicing positive self-talk(37.9\%) and spending more time with your family $(37.4 \%)$. Total number of factors which were selected by the participants were 10 . Number of factor (10) both gender who selected factors were same. Black participants (12) chose more factors than white participants (8). Only ethnicity was presented significant difference in following factors: Change in exercising $\left(\chi^{2}(1)=5.51, p=.019\right)$, Spending more time with family $\left(\chi^{2}(1)=10.202, p=.001\right)$, Developing a new hobby or reinvesting in previous hobbies $\left(\chi^{2}(1)=7.343, \mathrm{p}=.007\right)$, Practicing positive self-talk $\left(\chi^{2}(1)=10.20, \mathrm{p}=.001\right)$, and Addressing relationship or family issues/conflict $\left(\chi^{2}(1)=4.57\right.$, $\mathrm{p}=.032)$.

Figure 1. Average Drinks per Week \& Percent Change from Baseline.

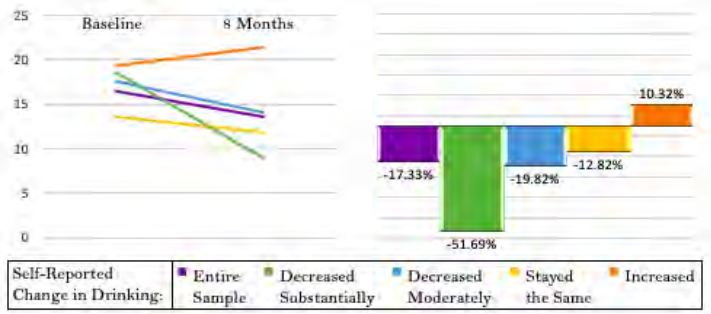


Figure 2. Over the past month has your overall drinking level changed. (self-reported).

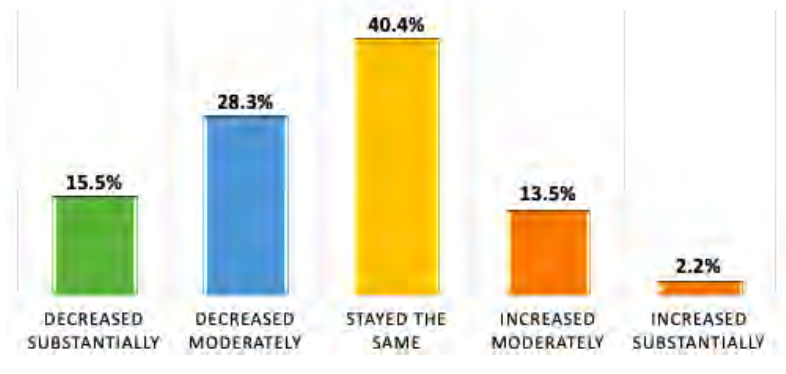

\section{Discussion}

The results of our study support findings from previous studies that demonstrate that young adults typically reduce their drinking without any formal treatment, which has been termed maturing out. Especially, actual drinking level over the past month from 8-month follow-up was an overtly reduced drinking level compared to self-report from the baseline. Ethnicity differences were explicitly shown by the different factors that were associated with either decreased or increased drinking. The only significant gender difference was for the factor change in financial situation, when associated with increased drinking. Change in financial situation could mean more money to buy alcohol or less money leading to more stress and drinking to cope. This could be consistent with prior studies showing financial problems promote excessive alcohol consumption (Khan et al., 2002, Peirce et al., 1994, Peirce et al., 1996). The provided results support that young adults generally reduce their drinking during their 20s, although drinking levels remained relatively high even after reductions and most still exceed NIAAA recommendation for moderate drinking.

The presented study sought to identify the specific factors that elicit either decreased or increased levels of drinking. The number of participants for increased drinking level was an inadequate sample's pool compared to the number of participants for decreased drinking level. Valid interpretation and generalization can only be given by a larger sample's pool size for increased drinking level.

Self-reported changes in the 8-months follow-up can be a debatable confound. Self-reported questionnaires, especially the question asking, "over the past month, has your overall drinking level changed," are greatly impacted by the participants' past week experience. For example, a participant had a party or celebration last week, then the drinking level is more likely to be increased. Contrastingly, another participant had a government exam or any official exam, then the drinking level is less than usual. Depending on the recent past event, responses can be given that do not truly reflect the participant's typical alcohol consumption.

Further research is needed to investigate how drinking behavior can be influenced if participants are given accurate feedback on their level of drinking and the efficacy of factors associated with changes in that target behavior.

\section{References}

Alvanzo, A. A., Storr, C. L., La Flair, L., Green, K. M., Wagner, F. A., \& Crum, R. M. (2011). Race/ethnicity and sex differences in progression from drinking initiation to the development of alcohol dependence. Drug and alcohol dependence, 118(2-3), 375-382.

Collins, R. L., Parks, G. A., \& Marlatt, G. A. (1985). Social determinants of alcohol consumption: The effects of social interaction and model status on the self-administration of alcohol. Journal of Consulting and Clinical Psychology, 53(2),

Dawson, D. A., Grant, B. F., Stinson, F. S., \& Chou, P. S. (2006). Maturing out of alcohol dependence: the impact of transitional life events. Journal of studies on alcohol, 67(2), 195-203.

Jackson, K. M., \& Sartor, C. E. (2016). The natural course of substance use and dependence. The Oxford handbook of substance use and substance use disorders, 1, 67-132.

Lee, M. R., \& Sher, K. J. (2018). "Maturing out" of binge and problem drinking. Alcohol research: current reviews, 39(1), 31.

Misch, D. A. (2007). " Natural recovery" from alcohol abuse among college students. Journal of American College Health, 55(4), 215-218.

Vik, P. W., Cellucci, T., \& Ivers, H. (2003). Natural reduction of binge drinking among college students. Addictive Behaviors, 28(4), 643-655.

Wilsnack, R. W., Wilsnack, S. C., Kristjanson, A. F., Vogeltanz-Holm, N. D., \& Gmel, G. (2009). Gender and alcohol consumption: patterns from the multinational GENACIS project. Addiction, 104(9), 1487-1500. 